\title{
Cellular automaton simulation of counter flow with paired pedestrians
}

\author{
Hui Xiong * \\ Department of Transportation Engineering, Beijing Institute of Technology \\ Beijing, 100081, China \\ E-mail: xionghui@bit.edu.cn \\ Xuedong Guo \\ Department of Transportation Engineering, Beijing Institute of Technology \\ Beijing, 100081, China \\ E-mail:05251127@bjtu.edu.cn \\ Wuhong Wang \\ Department of Transportation Engineering, Beijing Institute of Technology \\ Beijing, 100081, China \\ E-mail: wangwuhong@bit.edu.cn \\ Huachun Tan \\ Department of Transportation Engineering, Beijing Institute of Technology \\ Beijing, 100081, China \\ E-mail: tanhc@bit.edu.cn \\ Heng Wei \\ Department of Civil and Environmental Engineering, University of Cincinnati \\ Cincinnati, OH 45221-0071, USA \\ E-mail:weihg@ucmail.uc.edu \\ Received 13 July 2011 \\ Accepted 29 November 2011
}

\begin{abstract}
Knowledge on pedestrian behavior is the basis to build decision support system for crowd evacuation management in emergency. In this paper, the impact of paired walking behavior on pedestrian counter flow in a channel is studied. The pedestrian walking behaviors are simulated by the cellular automaton model and the pedestrians are classified as single right walker, single left walker, paired right walker, and paired left walker. Single walker can move forward, leftward, rightward or stand still. The paired pedestrians are considered as a combined unit similar to the single walker in terms of route choice and they can move to the same direction simultaneously. It is found that flow and velocity decrease with increase of the paired rate in case of stable density. Simulation results reveal the phase transitions in terms of density from free flow to the unstable flow and from the unstable flow to the congestion flow. However, the critical densities of phase transition are unaffected by the channel size.
\end{abstract}

Keywords: decision support system; cellular automaton; paired pedestrians; counter flow.

\footnotetext{
${ }^{*}$ Corresponding author. Tel.: +86-10-68915579; Fax: +86-10-68914582.

E-mail address: xionghui@bit.edu.cn.
} 


\section{Introduction}

Drivers and pedestrians are two main types of people in traffic system. Driving behavior has been studied by many researchers. ${ }^{1-4}$ Most focus on driver cognition, driving error, driving reliability and so on. In recent years, pedestrian flow modeling has drawn considerable attention, partly because the model serves as the basis for building decision support system for crowd evacuation management in emergency and pedestrian facility operations.

The existing pedestrian models can be classified as macroscopic models and microscopic models. Most macroscopic models are expressed in the form of partial differential equations, based on the principle of the conservation law in revealing relationships among pedestrian flow, density and speed. ${ }^{5,6}$ Due to the ability to reveal interactions among pedestrians, and between pedestrians and obstacles, micro-level pedestrian flow models are more widely applied. Typical models include cellular automaton models, ${ }^{7-13}$ lattice gas models, ${ }^{14-18}$ social force models, ${ }^{19}$ centrifugal force models, $^{20}$ and floor field models. ${ }^{21,22}$ In the cellular automaton (CA) models, walking space is set as a twodimensional system while being divided into cells. Each cell can be either empty, occupied by exactly one pedestrian, or occupied by wall or obstacle. At each time step, pedestrian is assumed to move to adjacent cells or stand still complying with the route choice rule and conflict avoidance rule.

Applying the cellular automaton technique, Blue and Adler (2001) simulated three bi-directional pedestrian flows, including flows in separated lanes, interspersed flow, and dynamic multilane flow. The results showed that the emergent fundamental parameters of speed, flow and density produced from the model were reasonable compared with the field data. ${ }^{7}$ Considering right-hand side walking preference, Fang et al (2003) studied bi-directional pedestrian movement. It was found that the critical density when phase transition occurred increased as the probability of back stepping rose, and the critical density declined with the increase of the system size. ${ }^{8}$ Weng et al (2006) simulated pedestrian counter flow with different walk velocities through update at different time step intervals. Simulation results revealed three phases of pedestrian patterns, including free movement phase, lane formation phase, and perfect stopping phase. ${ }^{9}$ Considering the impact of surrounding environment on pedestrian flow, $\mathrm{Yu}$ and Song (2007) introduced interaction radius parameter to route choice rules. It was found that the critical entrance density at the transition point did not depend on the system size when the radius was large. ${ }^{10}$ Applying a CA model, Yang et al (2008) introduced right-preference intensity to study pedestrian counter flow with right-moving preference. It concluded that right-preference was effective when the density was below critical value. ${ }^{11}$ Nagatani (2009) presented a bidirectional CA model for facing pedestrian flow on a wide passage. Results showed that the dynamical phase transitions occurred at three stages with increasing density. From the simulation, four traffic states were found, including the free traffic, jammed traffic 1, jammed traffic 2 , and frozen state. ${ }^{12}$ To study the effect of soft boundary on the pedestrian flow, Nagatani (2010) demonstrated that the dynamical phase transition had a strong dependence on the soft boundary. ${ }^{13}$

In this paper, a cellular automaton model without step back for pedestrian dynamics considering paired walking behavior is presented. The model reveals the influence of pair rates on pedestrian flow characteristics. The rest of the paper is organized with the following sections. Section II develops the cellular automaton model and defines walking rules for single and paired pedestrians. Section III applies the model to simulate pedestrian movement in various scenarios. The conclusions and recommendations for further study are presented in Section IV.

\section{Model}

The model is defined on a two dimensional system. The underlying structure is a $\mathrm{W} \times \mathrm{L}$ cell grid, where $\mathrm{W}$ is the width of the channel and $L$ is the length of the channel. Each cell can either be empty or occupied by wall, obstacle or exactly one pedestrian. The size of a cell is set to be $0.4 \times 0.4 \mathrm{~m}^{2}$. This is a typical space occupied by a person in a dense crowd. ${ }^{23}$ Each pedestrian can move only one cell per time step. In practice, the average velocity of a pedestrian is about $1.0 \mathrm{~ms}^{-1}$ under normal. ${ }^{24}$ So, the time step is set to be approximately $0.4 \mathrm{~s}$. 
Two types of the walkers are targeted in this study: walkers who are single and walkers who are paired with another one. For the single one, he/she is always single during the simulation, and for the paired pedestrians, they are always paired with the initial company. The channel is confined with walls as top and bottom boundaries. Any person can walk from left to right, named as the right walker; or from right to left, is called as the left walker. But no one can cross the top or bottom boundaries. The periodic boundary conditions are adopted in the model; in other words, if the right walker arrives at the right boundary, he/she moves in the system from the left boundary. If the left walker arrives at the left boundary, he/she moves in the system from the right boundary. The left and the right boundaries are close. Thus, the total number of walkers is conserved. Pedestrian movements within the channel are illustrated by Fig. 1.

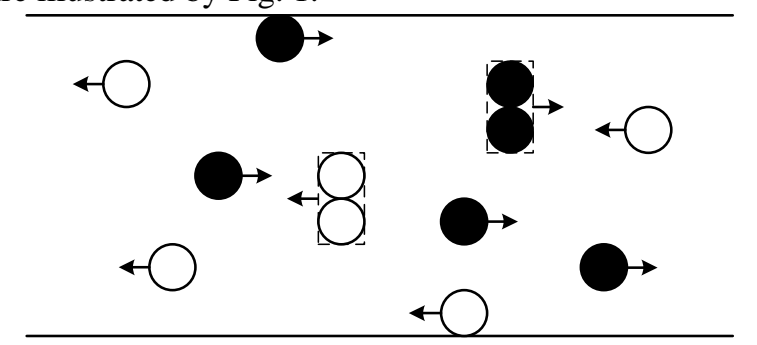

Fig. 1. Schematic illustration of the pedestrian counter-flow considering paired behavior in a channel. The black circle represents the right walker, and the white circle represents the left walker. The two in a dashed rectangle in black and in white represent the paired right walkers and the paired left walkers respectively.

The update is randomly sequential for all pedestrians at every time step. Fig. 2 shows all the possible configurations that a single right walker may encounter. In Fig. 2(a), the right adjacent cell is unoccupied, the right walker will select it to move into no matter whether the other adjacent cells are occupied or not. If the right cell is occupied at this time step, his route choice is dependent on the state of the upper cell and bottom cell. In Fig. 2(b), if both the upper cell and the bottom cell are free, the walker will select the upper cell or the bottom cell to move in with the probabilities of $\mathrm{P}_{\mathrm{L} 1}$ and $\mathrm{P}_{\mathrm{R} 1}$, where $\mathrm{P}_{\mathrm{L} 1}=\mathrm{P}_{\mathrm{R} 1}$. The walker can also select to be waiting for the next time step. In this case, the probability is set to be $\mathrm{P}_{\mathrm{W} 1}$. For configuration (c), the probability of selecting the bottom adjacent cell or waiting is $\mathrm{P}_{\mathrm{R} 2}$ and $\mathrm{P}_{\mathrm{W} 2}$, respectively. For configuration (d), the probability of selecting the upper adjacent cell or waiting is $\mathrm{P}_{\mathrm{L} 3}$ and $\mathrm{P}_{\mathrm{W} 3}$, respectively. For configuration (e), due to no step back is allowed, the walker has to wait at this time step with probability of $\mathrm{P}_{\mathrm{W} 4}$. Similarly, we can define the route choice rules for the possible configuration that the left walker may encounter.
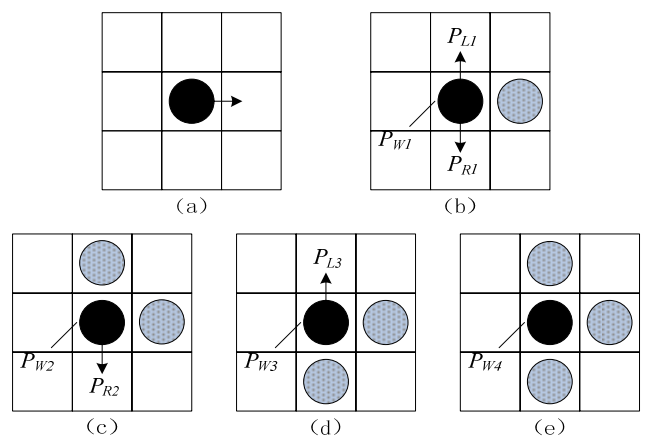

Fig. 2. All the possible configurations that a single right walker may encounter.

Fig. 3 shows all the possible configurations that paired walkers may encounter. The route choice rules for paired pedestrians are similar to those described above.

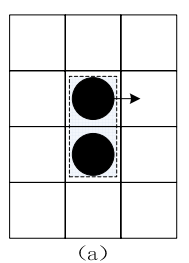

(a)

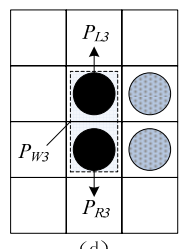

(d)
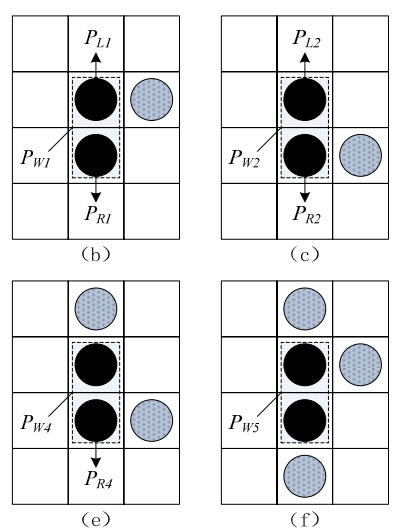

Fig. 3. All the possible configurations that paired walkers may encounter.

In Fig. 3(a), the two right adjacent cells are free, so the pair can move rightward simultaneously at this time step. In Fig. 3(b, c, d), if only one of the right cells is occupied or the both right cells are occupied, the pair will select moving up, down or staying still, and the corresponding probabilities are $\mathrm{P}_{\mathrm{L} 1}, \mathrm{P}_{\mathrm{R} 1}$ or $\mathrm{P}_{\mathrm{W} 1}$ for Fig. 3(b), $\mathrm{P}_{\mathrm{L} 2}, \mathrm{P}_{\mathrm{R} 2}$ or $\mathrm{P}_{\mathrm{W} 2}$ for Fig. 3(c), and $\mathrm{P}_{\mathrm{L} 3}, \mathrm{P}_{\mathrm{R} 3}$ or $\mathrm{P}_{\mathrm{W} 3}$ for Fig. 3(d). For configuration (e), the probability of selecting the bottom adjacent cell or waiting is $\mathrm{P}_{\mathrm{R} 4}$ and $\mathrm{P}_{\mathrm{W} 4}$, respectively. For configuration (f), due to no step back is allowed, the paired walkers have to wait at this time step with probability of $\mathrm{P}_{\mathrm{W} 5}$. Similarly, we can 
define the route choice rules for the possible configuration in which the left paired walkers may encounter.

\section{Simulation and Results}

Initially, the channel is set as $60 \times 60$. Pedestrians are distributed randomly in the channel and the number of the right walkers is equal to the number of the left walkers. The density of the system, $\langle d\rangle$, is defined as the ratio of the total pedestrians to the total cells. The mean flow $<\mathrm{f}>$ of pedestrians is calculated as the number of right walkers moved across the right boundary and the left walkers who moved across the left boundary at one time step. The mean velocity $\langle\mathrm{v}\rangle$ of pedestrians moving in one time step is defined as the number of the walkers moving forward, excluding those moving to the up, bottom or stand still, divided by the total number of pedestrians existing in the channel. For each simulation, total 150,000 time steps are carried out, and the value of $\langle\mathrm{d}\rangle,\langle\mathrm{f}\rangle$, and $\langle\mathrm{v}\rangle$ are calculated according to the results of the last 5,000 time steps. Ten times iterations are conducted for each simulation. We take the average of the 10 results as the final value.

As for the single walker, we set the probabilities as follows: $\mathrm{P}_{\mathrm{L} 1}=\mathrm{P}_{\mathrm{R} 1}=0.25, \quad \mathrm{P}_{\mathrm{W} 1}=0.5, \quad \mathrm{P}_{\mathrm{R} 2}=0.9, \quad \mathrm{P}_{\mathrm{W} 2}=0.1$, $\mathrm{P}_{\mathrm{L} 3}=0.9, \mathrm{P}_{\mathrm{W} 3}=0.1, \mathrm{P}_{\mathrm{W} 4}=1.0$. As for the paired walkers, the moving probabilities are set as $\mathrm{P}_{\mathrm{L} 1}=\mathrm{P}_{\mathrm{W} 1}=0.25$, $\mathrm{P}_{\mathrm{R} 1}=0.5, \quad \mathrm{P}_{\mathrm{R} 2}=\mathrm{P}_{\mathrm{W} 2}=0.25, \quad \mathrm{P}_{\mathrm{L} 2}=0.5, \quad \mathrm{P}_{\mathrm{L} 3}=\mathrm{P}_{\mathrm{R} 3}=0.25$, $\mathrm{P}_{\mathrm{W} 3}=0.5, \quad \mathrm{P}_{\mathrm{R} 4}=0.9, \quad \mathrm{P}_{\mathrm{W} 4}=0.1, \quad \mathrm{P}_{\mathrm{W} 5}=1.0$. All the probabilities we set here are according to paragraphs above and Refs. 25.

Fig. 4(a) shows the plots of the mean velocity against the density at various paired rates. At the very beginning, velocity remains stable with the increase of density. Then the value declines sharply to almost zero and stays constant. The upper stable velocity indicates fluent phase and the lower stable velocity represents jamming phase. The segment in between is the transitional stage. The critical density for the velocity changing from fluent phase to jamming phase varies with the paired rates. The higher the paired rate is, the lower the critical density will be.

Fig. 4(b) shows the plots of the mean flow rate against the density at various paired rates. With the increasing of the mean density, the flow rate is getting greater and greater. When the flow arrives at a critical point, it declines sharply to almost zero and remains stable with the increase of density. The flow before decrease represents the fluent phase and the flow remains stable indicates jamming phase. The segment in between is the transitional stage. Within this segment, flow declines with the increase of paired rates for a given density.

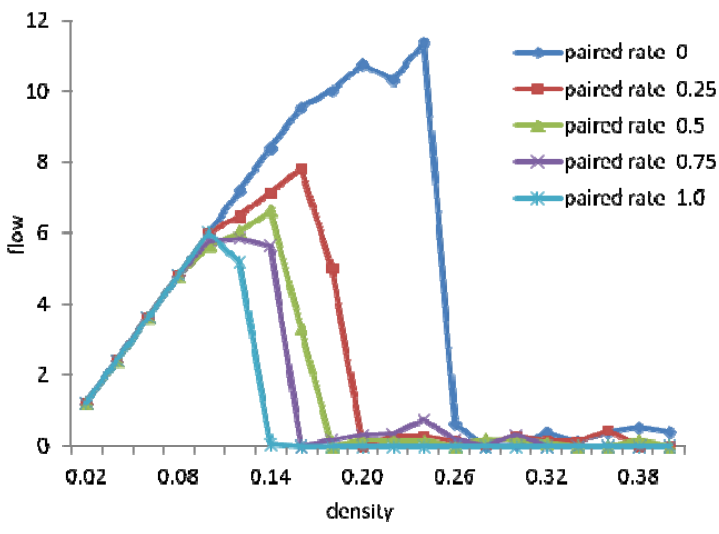

(a)

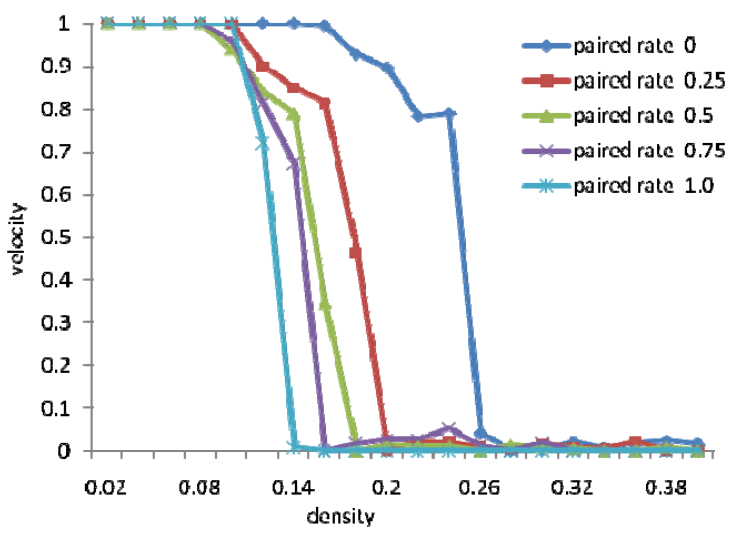

(b)

Fig. 4 . The flow rate $<\mathrm{f}>$ and velocity $<\mathrm{v}>$ against the density $<\mathrm{d}>$ at various paired rates.

Both Fig. 4(a) and Fig. 4(b) reflect the critical density from free flow to congestion. It is 0.1 without paired pedestrians, and 0.25 with all pedestrians paired in the paper.

Fig. 5(a) shows the relationships between flow rate and paired rates with various densities at $0.14,0.16$ and 0.18 . It is found that with the increase of the paired rates, the flow declines rapidly and arrives at approximately zero. The critical paired rate is 0.7 when density is 0.16 , and 0.4 when density is 0.18. Fig. 5(b) shows the similar characteristics compared with the plots in Fig. 5(a). We can conclude for a given density, the higher the paired rate is, the lower the flow or velocity will be. 


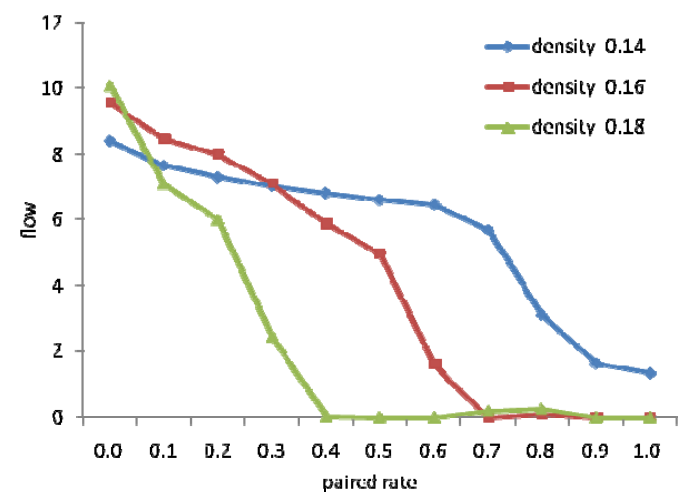

(a)

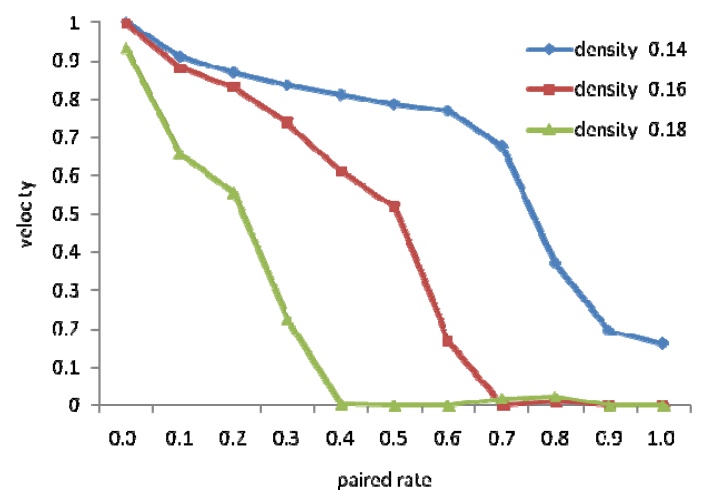

(b)

Fig. 5. The flow rate $<\mathrm{f}>$ and velocity $<\mathrm{v}>$ against paired rates with various densities.

When we enlarge the density gap, say the values of density are $0.08,0.24$, and 0.32 , we can reveal the characteristics of flow and velocity against paired rates when different densities are applied.

Fig. 6 (a) (b) shows the plots of the flow rate and velocity against the paired rates with larger density gaps. The flow rate and velocity remain maximal no matter what the paired rate is if the density is no more than 0.08 , and the flow rate and velocity approach zero no matter what the paired rate is if the density is no less than 0.32 .

In Fig. 7 we show the typical pattern of pedestrian movement at $\langle\mathrm{d}>=0.16$. The paired rates are set as 0.0 , $0.25,0.5,0.75$ for (a), (b), (c), and (d), respectively. When all the walkers are unpaired, see Fig. 7(a), they can move much more freely and the distribution of the pedestrians is uniform. When the paired rate is increasing, say 0.25 and 0.5 , more conflicts occurred, see Fig. 7(b) and (c). When the paired rate reaches 0.75 , the pedestrian flow is in a serious congestion, see Fig. 7(d).

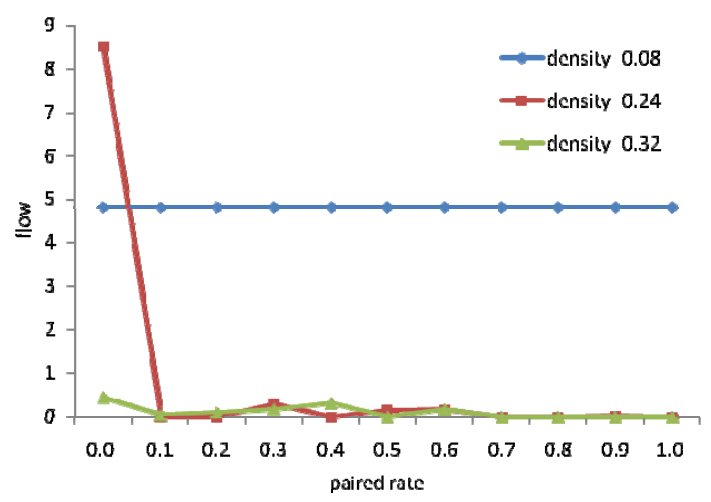

(a)

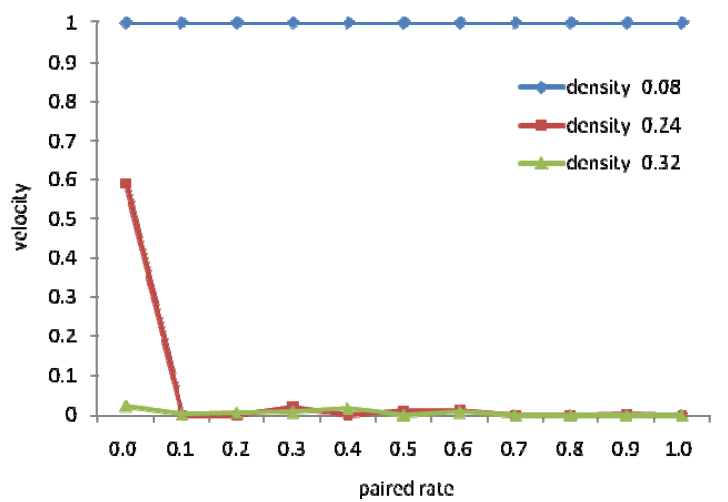

(b)

Fig. 6 . The flow rate $<\mathrm{f}>$ and velocity $<\mathrm{v}>$ against paired rates with larger density gaps

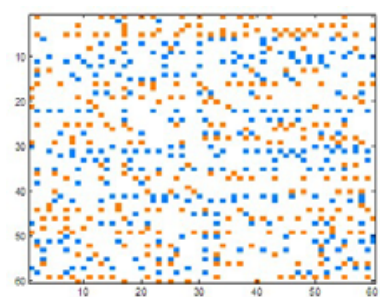

(a)

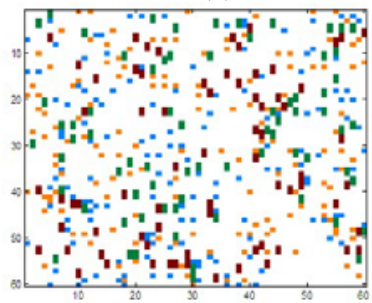

(c)

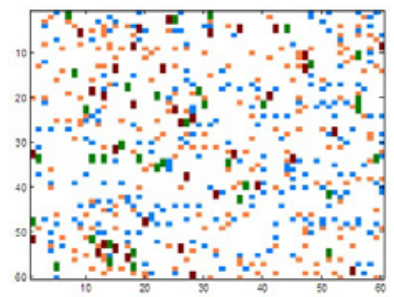

(b)

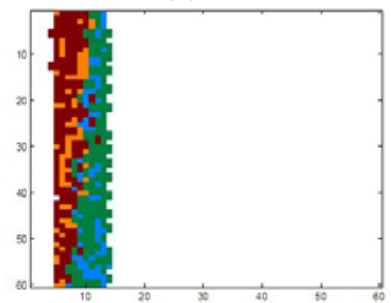

(d)
Fig. 7. The configurations of the paired pedestrians movement obtained at $\langle\mathrm{d}\rangle=0.16$. The cell in red, orange, green and blue represents the right paired pedestrian, the right single pedestrian, the left paired pedestrian and the left single pedestrian, respectively. 
To reveal the influence of channel size on pedestrian flow, we conduct simulation experiments with various channel sizes. Here we take $\mathrm{W} \times \mathrm{L}$ as $40 \times 40$, $50 \times 50,60 \times 60,70 \times 70$ and $80 \times 80$, respectively.

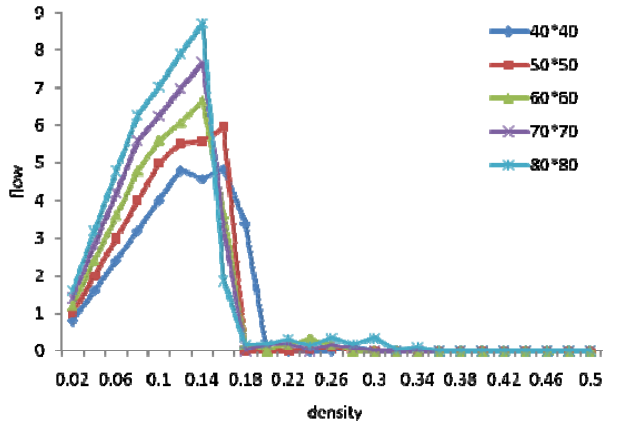

(a)

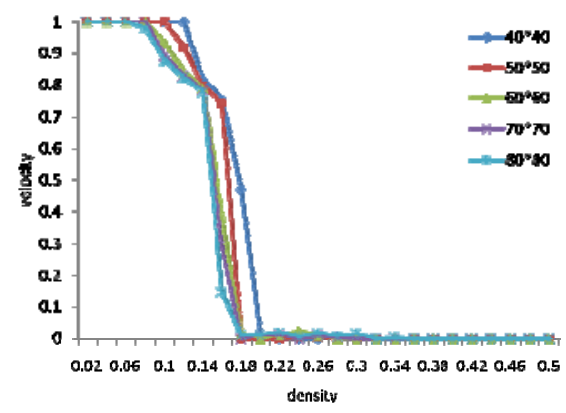

(b)

Fig. 8. The flow rate $<\mathrm{f}>$ against velocity $<\mathrm{v}>$ with various channel sizes. Here, the paired rate is set as 0.5 .

Fig. 8(a) shows the relationship between the flow rate and the density with various channel sizes. For a given density, the flow rate rises with the increase of channel size. However, the phase transition in terms of density from free flow to unstable flow or from unstable flow to jamming state remains almost unchanged. The critical density is not affected by the channel size. Fig. 8(b) shows the same flow characteristics in terms of relationships between velocity and density. Here, an interesting phenomenon is observed, that is, when the density is within the domain of $[0.08,0.18]$, the velocity declines with the increase of the channel size.

To further reveal the impact of channel size on flow characteristics, simulations are running for different channel sizes.

Fig. 9 shows the typical pattern of pedestrian movement at $\langle\mathrm{d}\rangle=0.16$ and paired $=0.6$. When the channel size is set as $40 \times 40$, the flow is at the state from free flow to unstable flow, see Fig. 9(a). When the channel size is set as $60 \times 60$, the flow is either at the unstable state or at the jamming state, see Fig. 9(b) and (c). This is the reason why the velocity declines with the increase of the channel size at some given densities. Fig. 9(d) shows the jamming flow when the channel size is set as $80 \times 80$. We explain this phenomenon as when the channel size increase, with the same density, conflicts within the channel increase and those conflicts may be distributed concentrated which can lower the moving efficiency and even cause congestion.

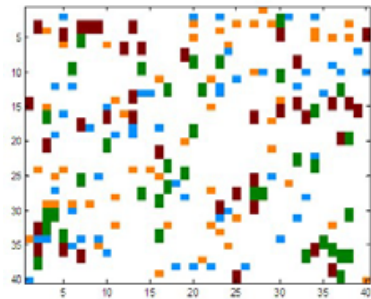

(a)

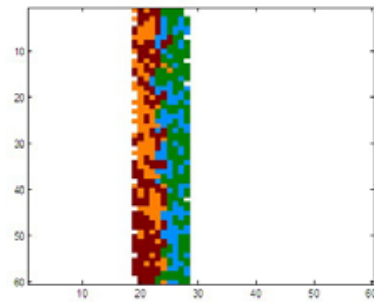

(c)

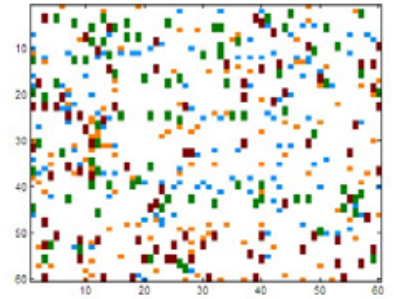

(b)

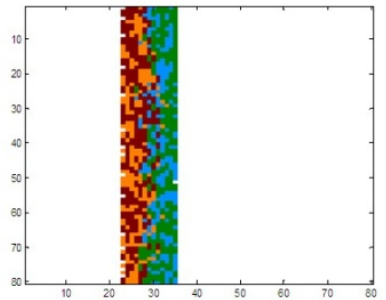

(d)

Fig. 9. The configurations of the paired pedestrians movement obtained at $\langle\mathrm{d}\rangle=0.16$ and paired rate $=0.6$. The channel sizes are set as $40 \times 40,60 \times 60,60 \times 60$ and $80 \times 80$ for (a), (b), (c), and (d), respectively. The cell in red represents the right paired pedestrian, and the orange cell represents the right single pedestrian, and the green cell represents the left paired pedestrian, and the blue cell represents the left single pedestrian.

\section{Conclusion}

This paper presents a study of impact analysis of paired walking behavior on pedestrian flow in a channel where the pedestrians are classified as single left walker, single right walker, paired left walker and paired right walker and their walking behaviors in the channel are simulated via a cellular automaton model.

The plots of flow or velocity against density reveal the phase transitions in terms of density from free flow to the unstable flow and from the unstable flow to the congestion flow. If we set the density as constant, flow and velocity decline with the increase of paired rates. To explore the influence of channel size on pedestrian flow, we simulate five scenarios with different channel 
sizes. Results show that the critical densities of phase transition are not affected by the channel size. The phenomenon that the velocity decreases with the increase of channel size due to a larger channel size always causes more conflicts with the same density, and when the conflicts are distributed concentrated congestion may form.

In the future work, some modifications of the model need to be done. For example, different walkers have different velocities, and under some circumstances, two paired pedestrians can walk separately for a while. As to the first case, we can designate a specific velocity to each pedestrian. For the second case, we can introduce probability to indicate the possibility that the paired pedestrians walk separately. In this situation, the separated pedestrians can be treated as two singles.

\section{Acknowledgements}

We acknowledge the support from Beijing Municipal Natural Science Foundation with Grant No. 8092026.

\section{References}

1. W.H. Wang, Q. Cao, K. Ikeuchi and H. Bubb, Reliability and safety analysis methodology for identification of drivers' erroneous actions, International Journal of Automotive Technology, 11(6) (2010) 873-881.

2. W.H. Wang, W. Zhang, H.W. Guo, H. Bubb and K. Ikeuchi, A safety-based approaching behavioural model with various driving characteristics, Transportation Research Part C-Emerging Technologies, 19 (2011) 1202-1214.

3. W.H. Wang, F.G. Hou, H.C. Tan and H. Bubb, A framework for function allocation in intelligent driver interface design for comfort and safety, International Journal of Computational Intelligence Systems, 3(5) 2010 531-541.

4. W.H. Wang, Y. Mao, J. Jing, X. Wang, H. W. Guo, X.M. Ren and K. Ikeuchi, Driver's various information process and multi-ruled decision-making mechanism: a fundamental of intelligent driving shaping model, International Journal of Computational Intelligence Systems, 4(3) (2011) 297-305.

5. R.L. Hughes, A continuum theory for the flow of pedestrians, Transp. Res., Part B: Methodol., 36 (2002) 507-535.

6. R. M. Colombo and M. D. Rosini, Pedestrian flows and non-classical shocks, Math. Meth. Appl. Sci., 28 (2005) 1553-1567.

7. V. J. Blue and J. L. Adler, Cellular automata microsimulation for modeling bi-directional pedestrian walkways, Transp. Res., Part B: Methodol., 35 (2001) 293-312.
8. W.F. Fang, L.Z. Yang, and W.C. Fan, Simulation of bidirection pedestrian movement using a cellular automata model, Physica A, 321 (2003) 633-640.

9. W. G. Weng, T. Chen, H. Y. Yuan, and W. C. Fan, Cellular automaton simulation of pedestrian counter flow with different walk velocities, Phys. Rev. E, 74(3) (2006) 036102.

10. Y. F. Yu and W. G. Song, Cellular automaton simulation of pedestrian counter flow considering the surrounding environment, Phys. Rev. E, 75(4) (2007) 046112.

11. L.Z. Yang, J. Li, and S.B. Liu, Simulation of pedestrian counter-flow with right-moving preference, Physica A, 387(13) (2008) 3281-3289.

12. T. Nagatani, Freezing transition in bi-directional CA model for facing pedestrian traffic, Phys. Lett. A, 373(33) (2009) 2917-2921.

13. T. Nagatani, Jamming and freezing transitions in CA model for facing pedestrian traffic with a soft boundary, Phys. Lett. A, 374 (2010) 1686-1689.

14. M. Muramatsu, T. Irie and T. Nagatani, Jamming transition in pedestrian counter flow, Physica A, 267 (1999) 487-498.

15. M. Muramatsu and T. Nagatani, Jamming transition of pedestrian traffic at a crossing with open boundaries, Physica A, 286 (2000) 377-390.

16. Y. Tajima, K. Takimoto, and T. Nagatani, Scaling of pedestrian channel flow with a bottleneck, Physica A, 294 (2001) 257-268.

17. Y. Tajima and T. Nagatani, Clogging transition of pedestrian flow in T-shaped channel, Physica A, 303 (2002) 239-250.

18. R. Jiang and Q. S. Wu, The moving behavior of a large object in the crowds in a narrow channel, Physica A, $\mathbf{3 6 4}$ (2006) 457-463.

19. D. Helbing and P. Molnar, Social force model for pedestrian dynamics, Phys. Rev. E, 51 (1995) 4282-4286.

20. W. J. Yu, R. Chen, L. Y. Dong and S. Q. Dai, Centrifugal force model for pedestrian dynamics, Phys. Rev. E, 72(2) (2005) 026112.

21. D. Yanagisawa and K. Nishinari, Mean-field theory for pedestrian outflow through an exit, Phys. Rev. E, 76(6) (2007) 061117.

22. H. J. Huang and R. Y. Guo, Static floor and exit choice for pedestrian evacuation in rooms with internal obstacles and multiple exits, Phys. Rev. E, 78(2) (2008) 021131.

23. C. Burstedde, K. Klauck, A. Schadschneider, and J. Zittartz, Simulation of pedestrian dynamics using a twodimensional cellular automaton, Physica A, 295 (2001) 507-525.

24. D. Helbing, L. Farkas, and T. Vicsek, Simulating dynamical features of escape panic, Nature, 407 (2000) 487-490.

25. J. Li, L.Z. Yang and D.L. Zhao, Simulation of bidirection pedestrian movement in corridor, Physica A, 354 (2005) 619-628. 